\title{
Ideias, un modelo de evaluación para inclusión digital y alfabetización informacional orientado a salud
}

\author{
Por Aurora Cuevas-Cerveró y María-Antonia García-Moreno
}

\begin{abstract}
Resumen: El principal propósito del proyecto Ideias (Inclusión digital y educación informacional para salud), ha sido mejorar los procesos de inclusión digital en el nordeste de Brasil mediante la formación y el avance tecnológico. Se presentan los primeros resultados del proyecto: un modelo de evaluación aplicado a los Agentes comunitarios de salud (ACS) del Estado de Sergipe, una figura de gran importancia en el Sistema Único de Salud (SUS) de Brasil. La propuesta involucra destrezas, conocimientos y actitudes con un interés educativo. En el modelo se mezclan y entretejen la educación en salud, la educación para la información, la formación tecnológica y la ética.
\end{abstract}

Palabras clave: Alfabetización informacional, Inclusión digital, Evaluación, Salud, Agentes comunitarios de salud.

Title: Ideias, an evaluation model for digital inclusion and information literacy with a focus on health

Abstract: The project objective for Ideias (Digital inclusion and informational education for health) has been to improve the processes of digital inclusion in the Northeast of Brazil through training and technological development. The first results of the project include an evaluation model applied to the Community health agents (ACS) of the state of Sergipe, an entity of great importance in the Universal Health System (SUS) in Brazil. The proposed model involves skills, knowledge and attitudes with an educational interest, which the model mixes and interweaves in topics such as health, education for information, technology training and ethics.

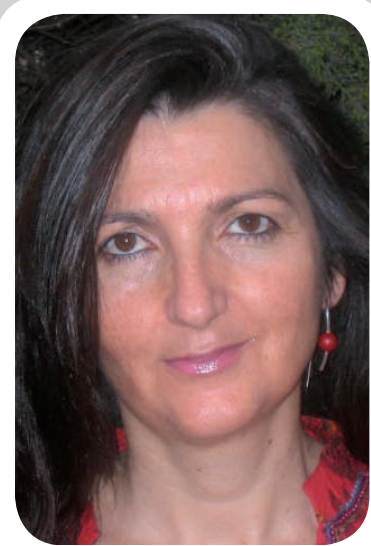

Aurora Cuevas-Cerveró, doctora en documentación y licenciada en filosofía, es profesora en la Facultad de Ciencias de la Documentación de la Universidad Complutense de Madrid (UCM). Miembro del grupo de investigación Biblioteca y Sociedad (Bisoc) de la UCM, sus publicaciones y actividad investigadora se centran en el estudio de la lectura, alfabetización en información, inclusión digital y competencia lectora en los nuevos entornos, con especial atención a las bibliotecas educativas.

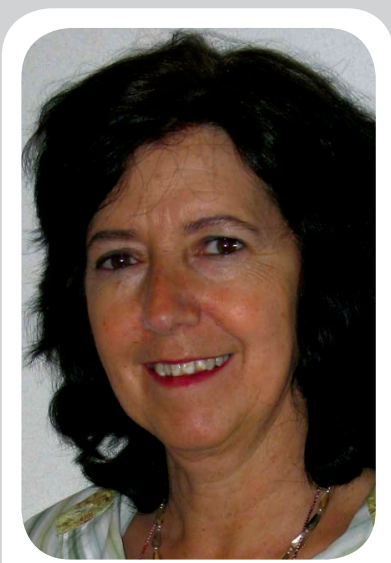

María-Antonia García-Moreno es profesora de la Facultad de Ciencias de la Documentación de la Universidad Complutense de Madrid. Entre sus líneas de investigación destacan las bases de datos, las bibliotecas digitales, arquitectura de la información, diseño de sistemas de información y gestión documental y bibliotecas multiculturales. Es miembro del grupo de investigación Bisoc (Biblioteca y Sociedad) y trabaja en proyectos de alfabetización informacional e inclusión digital.

Keywords: Information literacy, Digital inclusion, Evaluation, Health, Community health agents.

Cuevas-Cerveró, Aurora; García-Moreno, María-Antonia. “Ideias, un modelo de evaluación para inclusión digital y alfabetización informacional orientado a salud”. El profesional de la información, 2010, mayo-junio, v. 19, n. 3, pp. 240245.

DOI: 10.3145/epi.2010.may.03

\section{Introducción}

La elaboración de una propuesta que ayudara a medir el grado de inclusión digital y alfabetización informacional de los Agentes comunitarios de salud (ACS) brasileños, una figura de gran importancia en el Sistema Único de Saúde (SUS) de Brasil, ha sido el objetivo central del proyecto Ideias (Inclusión digital y educación informacional para salud), que durante el año 2009 ha trabajado paralelamente con otro proyecto brasileño de inclusión digital: Projeto de inclusão digital dos agentes comunitários de saúde em Sergipe ${ }^{1}$.
Ideias no busca únicamente medir la eficacia del aprendizaje en el proceso de inclusión digital, sino que pretende también servir como patrón en el diseño del propio programa de aprendizaje. Cumple así una doble función: a partir del modelo se programan los contenidos para más tarde servir en la medición del nivel de competencias obtenidos.

Brasil posee un sistema público sanitario: Sistema Único de Salud (SUS) considerado por la Organización Mundial de la Salud ${ }^{2}(O M S)$ como uno de los mayores del mundo, cuyas acciones no sólo cubren la atención 
primaria, de urgencia y hospitalaria, sino que dedica especial atención y recursos a la prevención mediante campañas (como la más reciente para combatir el dengue), y a la formación, tanto de los ciudadanos como del personal sanitario. Fue creado en 1988 por la Constitución federal brasileña, afianzado en 1990 por la Ley orgánica de salud y en la actualidad se dirige a 180 millones de brasileños.

\section{"Brasil cuenta con 229.900 ACS que atienden a 113,6 millones de personas (59,9\% de la población)"}

Según datos del Ministerio de Salud brasileño, el país cuenta con 229.900 ACS que atienden a 113,6 millones de personas, lo que representa el 59,9\% de la población. Los agentes actúan dentro de los equipos del Programa de salud de familia (PSF) para mejorar la atención básica. Son mediadores entre los habitantes de las distintas comunidades y los profesionales sanitarios. El PSF está presente en el $94 \%$ de los municipios del país, atendiendo al 49,9\% de la población brasileña. Un total de 29.710 equipos actúan en 5.229 municipios, siendo la región del nordeste la que tiene un mayor porcentaje de cobertura $(69,9 \%)$, seguido de centro/oeste y norte $(49,4 \%)$, sur $(48,5 \%)$ y sudeste $(37,3 \%)$ (gráfico $1)$.

http://portal.saude.gov.br

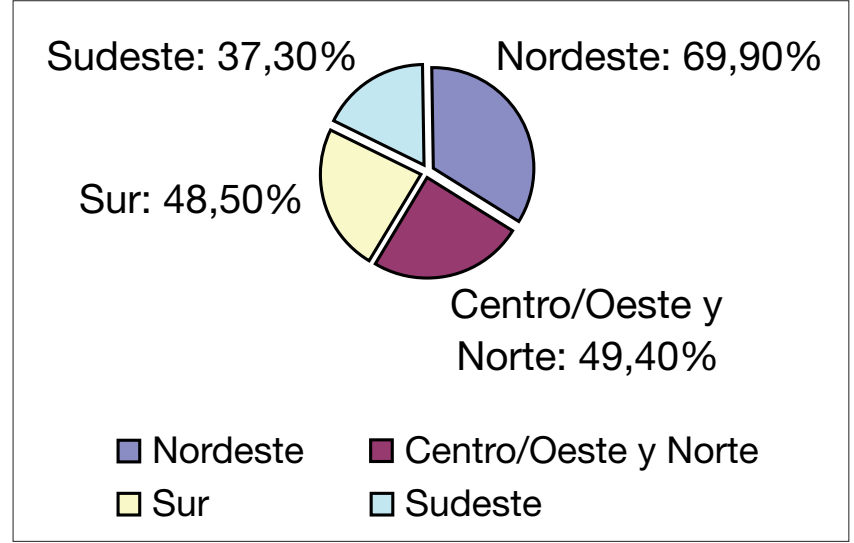

Gráfico 1. Cobertura del programa de salud de familia Fuente: Ministerio de Salud (Brasil)

Ideias se ha integrado en un proyecto de inclusión digital dirigido a $A C S$ que trabajan en un $P S F$ en 13 de los 75 municipios del estado de Sergipe, en el nordeste de Brasil, y que tiene entre sus objetivos principales el fortalecimiento de las actividades de prevención sanitaria y de promoción de la salud.
En el mismo han participado 750 agentes de los 10.276 que conforman el grupo de la región de Sergipe. Lo han hecho mediante talleres de informática, alfabetización digital y cursos a distancia (online) sobre aspectos de salud y otros de carácter divulgativo. Se ha utilizado el modelo Ideias, basado en competencias y en los indicadores que permiten valorar la propuesta de inclusión digital y educación informacional para salud.

\section{Los agentes comunitarios de salud}

En 1991 el Ministerio de Salud de Brasil, implantó en todo el país el Programa de agentes comunitarios de salud (PACS) con una idea de cambio en la concepción de la asistencia pública sanitaria, y en 1994 creó el Programa de salud de la familia (PSF) como estrategia del modelo de atención básica dentro del SUS.

Los ACS son considerados profesionales por el $\mathrm{Mi}$ nisterio de Salud brasileño, aunque con unas características especiales: deben residir en la misma comunidad en la que trabajan, ser mayores de dieciocho años y tener disponibilidad de tiempo para llevar a cabo las actividades requeridas. Trabajan con familias de áreas geográficas bien definidas, se encargan de la inscripción y seguimiento de las mismas, realizan entrevistas, hacen visitas a domicilio y organizan reuniones con la comunidad.

La función que cumplen los ACS como mediadores entre la población y el sistema sanitario transciende más allá del registro de enfermedades, ya que entre sus cometidos se encuentra la prevención de enfermedades en los niños, mujeres y ancianos, entre otros grupos. Hay trabajos que demuestran cambios importantes en algunas regiones de Brasil respecto a períodos en los que no existían los $A C S$, sobre todo, en cuanto a la mortalidad infantil y enfermedades de la infancia (Cesar, 2002). Todavía hoy se trabaja en un programa para la reducción de la mortalidad infantil que aún es muy elevada en el nordeste del país, siendo el 93,9\% de los casos de muerte producidos por diarreas.

http://portal.saude.gov.br

\section{"Los ACS deben residir en la misma comunidad en la que trabajan, ser mayores de 18 años y disponer de tiempo"}

Los ACS tienen una fuerte presencia en el nordeste de Brasil desde hace más de 20 años. Promueven prácticas saludables en la familia y en su comunidad, en coordinación con el personal de salud. No son per- 
sonal sanitario especializado pero su función social es altamente favorable para asegurar la salud de la familia. Transmiten, por ejemplo, información y recomendaciones sobre dietas en casos de trastornos como diabetes, deshidratación infantil y cuidados de la hipertensión o higiene sexual, entre otros. A estos Agentes se ha dirigido el programa formativo del proyecto Ideias.

\section{Ideias}

Ideias $^{3}$ y el citado proyecto interdisciplinar de la Universidad de Brasilia, Projeto de inclusão digital dos agentes comunitários de saúde em Sergipe, van unidos en su planteamiento.

Ideias, basado en competencias digitales, informacionales y sociales, es un modelo evaluativo especialmente indicado para el aprendizaje permanente. Además de valorar la eficacia del proceso educativo de los $A C S$ permite intervenir en el diseño de los programas añadiendo las competencias informacionales y lectoras estrechamente ligadas a las digitales.

Nuestro interés radica en contribuir a mejorar la calidad de los aprendizajes en entornos no formales de enseñanza y contrastar la validez que presentan en un contexto socio-económico desfavorable y en un aspecto altamente vulnerable como es la salud pública.

Se ha llevado a cabo durante el año 2009 en Sergipe, pequeño estado del nordeste de Brasil, sobre los ACS de 42 municipios que atienden a 938.912 personas, por lo general, integrantes de comunidades desfavorecidas.

Aunque la formación se planteó inicialmente para que se impartiese en cuatro de las bibliotecas públicas, finalmente y debido a dificultades de infraestructura, se llevó a cabo en los Centros de apoyo tecnológico para inclusión social (Catis) de los municipios de Itabaiana, Lagarto, Propriá y Tobias Barreto, donde se concentraba el mayor número de ACS. El Ministério de Ciência y Tecnología de Brasil dota a los telecentros de la infraestructura necesaria para la realización de cursos.

\section{"Los ACS promueven prácticas saludables en coordinación con el personal de salud de la comunidad"}

El modo de trabajo fue colaborativo. El equipo de investigación de la Universidad Complutense aportó el modelo teórico, la programación didáctica y el sistema de indicadores para el seguimiento y valoración de re- sultados. El equipo brasileño se encargó de la adaptación contextual del modelo propuesto a las realidades concretas del nordeste de Brasil y se ocupó de la ejecución del programa, garantizando su cumplimiento.

El trabajo consistió en una categorización de competencias digitales e informacionales para la inclusión digital de los ACS. Como base teórica se tuvieron en cuenta trabajos precedentes del equipo y se revisó la literatura científica nacional e internacional, con énfasis en el caso brasileño. Concluimos con un esquema general que sirvió de base a la producción de indicadores.

El modelo general se estructuró en cuatro partes, las tres primeras se adecuan a un esquema de destrezas, conocimientos y actitudes y la cuarta pretende medir a largo plazo el impacto social del programa en la salud de la comunidad: inclusión digital, inclusión informacional, inclusión social e impacto social.

Los grupos de indicadores se relacionan de forma estructural conformando un modelo holístico que integra en un mismo orden de categorías las dimensiones digital, informacional y social. Esto exige que cada indicador esté relacionado en la estructura con todos los de su categoría e iguala a los indicadores en su nivel de importancia en el conjunto.

Además de medir la eficacia del nivel de aprendizaje, Ideias funciona como patrón en el diseño del programa de aprendizaje, es decir, partiendo del modelo se programan los contenidos y al final del proceso sirven para medir el nivel de competencias logrado.

El primer resultado incluye 3 grandes tópicos ${ }^{4}, 18$ parámetros y 54 indicadores que, a su vez, están desglosados en competencias que constituyen criterios de valoración.

\section{"El trabajo consistió en una categorización de competencias digitales e informacionales para la inclusión digital de los ACS"}

Para la formación de los ACS el Projeto de inclusão digital dos agentes comunitários de saúde em Sergipe se ocupó de la formación de los formadores que actuarían como mediadores en el proceso. Esta instrucción previa, de tipo presencial, se realizó desde julio a septiembre de 2009 e incluyó informática básica y software libre: GNU/Linux, adaptación de ambiente de trabajo, BrOffice.org.Impress, Broffice.org.Calc, BrOffice.org.Draw, Designer gráfico básico: GIMP e Scri- 


\begin{tabular}{|c|c|c|}
\hline Destrezas & Conocimientos & Actitudes \\
\hline $\begin{array}{l}\text {-I- } \\
\text { Inclusión digital } \\
\text { Alfabetización digital } \\
\text { Se centra en las competencias } \\
\text { digitales }\end{array}$ & $\begin{array}{l}\text {-II- } \\
\text { Inclusión informacional } \\
\text { Alfabetización informacional } \\
\text { Se centra en las competencias informaciona- } \\
\text { les }\end{array}$ & $\begin{array}{l}\text {-III- } \\
\text { Inclusión social } \\
\text { Se centra en el aprendizaje para la } \\
\text { evolución de los ciudadanos: inte- } \\
\text { gración social de los ciudadanos al- } \\
\text { fabetizados digital e informacional- } \\
\text { mente }\end{array}$ \\
\hline Acceso a las TIC & Acceso a la información & Autonomía en el aprendizaje \\
\hline $\begin{array}{l}\text { - Acceso y distinción del soft- } \\
\text { ware ofimático } \\
\text { - Manejo de las aplicaciones de } \\
\text { acceso a internet } \\
\text { - Manejo de las herramientas de } \\
\text { búsqueda y recuperación de in- } \\
\text { formación }\end{array}$ & $\begin{array}{l}\text { - Reconocimiento de la necesidad de infor- } \\
\text { mación } \\
\text { - Identificación de fuentes de información } \\
\text { - Uso de estrategias para la localización de } \\
\text { información }\end{array}$ & $\begin{array}{l}\text { - Consultas en bibliotecas virtuales } \\
\text { - Consultas en recursos especializa- } \\
\text { dos en la Red } \\
\text { - Lectura de documentos digitales } \\
\text { especializados en red }\end{array}$ \\
\hline Uso de las TIC & Uso de la información & Uso efectivo de redes sociales \\
\hline $\begin{array}{l}\text { - Gestores de contenidos (blogs, } \\
\text { wikis...) } \\
\text { - Selección de canales y alertas } \\
\text { de información especializada } \\
\text { - Plataformas de enseñanza- } \\
\text { aprendizaje (e-learning) }\end{array}$ & $\begin{array}{l}\text { - Organización de la información } \\
\text { - Integración de nueva información al acervo } \\
\text { personal } \\
\text { - Aplicar la información incorporada para la } \\
\text { resolución de problemas }\end{array}$ & $\begin{array}{l}\text { - Frecuencia de consultas relaciona- } \\
\text { das con salud en la red } \\
\text { - Variedad en los recursos utiliza- } \\
\text { dos. } \\
\text { - Adscripción a colectivos relaciona- } \\
\text { dos con salud en la red (asociacio- } \\
\text { nes, foros de discusión, listas de } \\
\text { distribución, redes sociales, etc.) }\end{array}$ \\
\hline Lectura digital & Lectura de la información & Comunicación con instituciones \\
\hline $\begin{array}{l}\text { - Habilidades para navegar en } \\
\text { textos con hipervínculos } \\
\text { - Lectura en dispositivos electró- } \\
\text { nicos diversos } \\
\text { - Uso de sistemas de búsqueda } \\
\text { avanzada }\end{array}$ & $\begin{array}{l}\text { - Comprensión de la información } \\
\text { - Lectura en distintos formatos y soportes } \\
\text { - Lectura con distintas finalidades (ocio, } \\
\text { aprendizaje, trabajo) }\end{array}$ & $\begin{array}{l}\text { - Conocimiento de la red sanitaria } \\
\text { - Conocimiento y uso de documen- } \\
\text { tos institucionales relacionados } \\
\text { con salud (folletos, informes, es- } \\
\text { tudios, etc.) } \\
\text { - Conocimiento de programas y ac- } \\
\text { tividades sanitarias }\end{array}$ \\
\hline Redes digitales & Producción y comunicación de la información & Comunicación (social e intercultural) \\
\hline $\begin{array}{l}\text { - Uso de redes sociales en el ám- } \\
\text { bito de la salud } \\
\text { - Habilidades para el trabajo en } \\
\text { colaboración } \\
\text { - Participación en comunidades } \\
\text { virtuales de aprendizaje }\end{array}$ & $\begin{array}{l}\text { - Comunicación de la información } \\
\text { - Ccooperación en la producción de conoci- } \\
\text { miento } \\
\text { - Generación de nuevo conocimiento (a par- } \\
\text { tir de la información incorporada y apre- } \\
\text { hendida) }\end{array}$ & $\begin{array}{l}\text { - Elaboración de información básica } \\
\text { sanitaria } \\
\text { - Difusión de información sanitaria } \\
\text { - Organización de actividades (char- } \\
\text { las, conferencias, talleres, etc.) }\end{array}$ \\
\hline Evaluación de las TIC & Evaluación de la información & Actitud crítica ante la información \\
\hline $\begin{array}{l}\text { - Facilidad de acceso y conectivi- } \\
\text { dad } \\
\text { - Uso de las TIC } \\
\text { - Actualización y mantenimiento } \\
\text { de las TIC }\end{array}$ & $\begin{array}{l}\text { - Búsqueda } \\
\text { - Calidad de los contenidos } \\
\text { - Fuentes de información }\end{array}$ & $\begin{array}{l}\text { - Reconocimiento de la procedencia } \\
\text { y calidad de los recursos } \\
\text { - Uso de recursos de reconocido } \\
\text { prestigio }\end{array}$ \\
\hline Ética de las TIC & Ética de la información & Compromiso social \\
\hline $\begin{array}{l}\text { - Uso responsable de las TIC } \\
\text { - Uso de tecnología de código } \\
\text { abierto } \\
\text { - Compartir habilidades tecnoló- } \\
\text { gicas con la comunidad }\end{array}$ & $\begin{array}{l}\text { - Interés por la información en toda su diver- } \\
\text { sidad (contexto, fuentes y culturas diver- } \\
\text { sas) } \\
\text { - Respeto de los derechos de libertad y pro- } \\
\text { piedad intelectual } \\
\text { - Compartir información con la comunidad }\end{array}$ & $\begin{array}{l}\text { - Pertenencia a asociaciones de sa- } \\
\text { lud } \\
\text { - Participación activa en la red } \\
\text { - Activismo en red }\end{array}$ \\
\hline
\end{tabular}

Tabla 1. Indicadores para inclusión digital e informacional orientada a salud. Modelo holístico. Fuente: Resultados de la Fase 2 del proyecto Ideias

bus, introducción a Moodle, comunicación comunitaria (webradio, e-cine, blog, wiki y vídeo) y finalmente, montaje y mantenimiento de redes.
La formación continuó en octubre de 2009 y fue realizada mediante talleres online, utilizando recursos en tecnologías inclusivas con software libre. Los con- 
tenidos se centraron en competencias informacionales, educación y comunicación en salud, familia y comunidad. Un mes después se distribuyó a los ACS el instrumento que Ideias había creado para la evaluación.

\section{http://164.41.105.58/inclusaodigitalmoodle/}

Para probar los cuestionarios participaron en este taller inicial 35 de los 750 ACS involucrados en el proyecto de inclusión digital, de tal manera que hicieran posible los ajustes necesarios, tanto en el programa de formación como en el instrumento ${ }^{5}$. En diciembre de 2009 este taller se puso a disposición de la totalidad de los ACS dentro de la muestra de los 4 municipios inicialmente escogidos para este análisis.

Los contenidos centrados en alfabetización informacional presentan el concepto de alfabetización en información y alfabetización digital unidos al de comunicación en salud, creando un nuevo concepto alfabetización digital en información y comunicación en salud. Se procuró acercar y hacer visible a los ACS la importancia de las competencias adquiridas para su práctica profesional diaria. Los contenidos están organizados en cuatro módulos: aprender a investigar, aprender a escoger, aprender a comunicar y ética de la comunicación. El cuestionario fue puesto a disposición de los agentes en formato digital y accesible online para que voluntariamente respondiesen a las preguntas propuestas.

La encuesta ${ }^{6}$, elaborada a partir de los 54 indicadores del modelo Ideias, propone preguntas sobre tres grandes áreas del proceso de inclusión digital en salud: inclusión digital, inclusión informacional e inclusión social. Las cuestiones relativas a la ética se convierten en un tema transversal a todas estas áreas y permitieron a los ACS llevar a cabo una reflexión sobre la base de que las acciones de inclusión digital deben ser construidas para posibilitar un aprendizaje efectivamente democrático, interactivo, colectivo y colaborativo.

\section{"Las competencias digitales e informacionales pueden mejorar los resultados de las campañas lanzadas por el Ministerio de Salud de Brasil"}

\section{Conclusiones}

Los ACS, como educadores en salud en comunidades desfavorecidas, son un grupo de gran interés para trabajar con ellos en procesos de alfabetización digital e informacional.
El modelo evaluativo que propone Ideias involucra destrezas, conocimientos y actitudes con una clara vocación educativa, y en él se mezclan y entretejen la educación en salud, la educación para la información y la formación tecnológica.

La capacidad de aprendizaje continuo que promueven las competencias digitales e informacionales pueden contribuir positivamente a mejorar los resultados de las campañas lanzadas por el Ministerio de Salud de Brasil (combate del dengue o pacto contra la mortalidad infantil, entre otras) que pueden ser extrapolable a otras comunidades o países.

Las cuestiones relativas a la ética se muestran de modo transversal tanto en los contenidos como en los indicadores porque entendemos que las acciones de inclusión digital deben ser construidas para posibilitar un aprendizaje efectivamente democrático, interactivo, colectivo y colaborativo.

Ideias establece expresamente en su planteamiento el impacto social, la posibilidad de transformación de los colectivos implicados. Se cuida sobre todo la dimensión ética: ética de la información, uso responsable de las TIC y compromiso social con la comunidad para contribuir sosteniblemente a su evolución.

\section{"Ideias establece expresamente en su planteamiento el impacto social, o sea, la posibilidad de transformación de los colectivos implicados"}

\footnotetext{
Notas

1. Financiado por la Secretaría de Ciência e Tecnologia para Inclusão Social del Ministério de Ciência e Tecnologia de Brasil.

2. Organización Mundial de la Salud. Informe sobre la salud en el mundo, 2008.

http://www.who.int/whr/2008/es/index.html
}

3. El proyecto Ideias ha sido diseñado por un grupo de investigación de la Facultad de Ciencias de la Documentación de la Universidad Complutense y financiado por la Agencia Española de Cooperación Internacional para el Desarrollo.

4. Hemos omitido los indicadores de impacto social en estos primeros resultados porque entendíamos que no podíamos medir esta parte hasta que no hubiera transcurrido un determinado período de tiempo que permitiera a los ACS aplicar en su comunidad los conocimientos adquiridos.

5. El cuestionario fue aplicado en dos momentos: antes y después de los talleres formativos. Fue previamente sometido a una adaptación contextual que actuara con mayor efectividad en la realidad de Sergipe. Esta adaptación contextual puede consultarse en el informe técnico: Cuevas-Cerveró, Aurora (coord.). Modelo teórico de evaluación: informe técnico del proyecto Ideias. Edición del grupo de investigación participante en el proyecto Ideias. Depositado en la biblioteca de la Facultad de Ciencias de la Documentación de la Universidad Complutense.

6. Puede consultarse el cuestionario completo en: Cuevas-Cerveró, Aurora 
(coord.). Afabetización informacional e inclusión digital: hacia un modelo de infoinclusión social. Gijón: Trea, 2010 [en prensa].

\section{El proyecto}

El proyecto Ideias ha sido financiado por Resolución de 17 de diciembre de 2008 de la presidencia de la Agencia española de cooperación internacional para el desarrollo (Aecid), Programa de cooperación interuniversitaria e investigación científica entre España e Iberoamérica. A/017156/08. En el proyecto Ideias han participado investigadores de la Universidad de Brasilia y de la Universidad Complutense de Madrid.

\section{Bibliografía}

Cesar, Juraci A.; Cavaleti, Marcelo A.; Holthausen, Ricardo S.; De-Lima, Luis-Gustavo S. "Mudanças em indicadores de saúde infantil em um município com agentes comunitários: o caso de Itapirapuã Paulista, Vale do Ribeira, São Paulo, Brasil". Cadernos de Saúde Pública, 2002, nov-dic, v. 18, n. 6. http://www.scielo.br/scielo.php?script $=$ sci_arttext\&pid=S0102-311X2002 $000600019 \& \ln g=e n \& n r m=i s o \& \ln g=p t$

Cuevas-Cerveró, Aurora (coord.). Modelo teórico de evaluación: informe técnico del proyecto Ideias. Agencia española de cooperación internacional para el desarrollo. Depositado en la Biblioteca de la Facultad de Ciencias de la Documentación de la Universidad Complutense de Madrid, 2009.

Cuevas-Cerveró, Aurora; Simeão, Elmira (Coord.). Alfabetización informacional e inclusión digital: hacia un modelo de infoinclusión social. Gijón: Trea, 2010 [en prensa]

Cuevas-Cerveró, Aurora; Simeão, Elmira. "Ideias, un modelo holístico de inclusión digital e informacional orientado a salud". Educación y biblioteca, 2009, v. 21, n. 172, pp. 105-107.

Garabeli-Cavalli-Kluthcovsky, Ana-Cláudia; Magosso-Takayanagui, Angela-Maria. "Agente comunitario de salud: una revisión de literatura". Revista Latino-americana de Enfermagem, 2006, novembro-dezembro, v. 14, n. 6. http://www.scielo.br/scielo.php? pid=S0104-11692006000600019\&script $=$ sci_arttext\&tlng=es

López-López, Pedro; Samek, Toni. "Inclusión digital: un nuevo derecho humano". Educación y biblioteca, 2009, v. 21, n. 172, pp. 114-118. http://dialnet.unirioja.es/servlet/fichero_articulo?codigo $=3026328 \&$ orden $=0$

Mendonça, Ana-Valéria; Sousa, Maria-Fátima; Nunes-Linhares, Ronaldo; Cabral, Júlio-César; Boaventura-Sales-Paixao, Pablo. "Inclusão digital dos agentes comunitários de saúde no Brasil: novas formas de aprender em rede". Em: Tempus-actas de saúde coletiva, 2009, v. 3, n. 1, pp. 28-37. http://www.tempusactas.unb.br/index.php/tempus/article/viewFile/707/714

Organización Panamericana de la Salud. Guía para facilitadores de la capacitación a agentes comunitarios de salud. Componente comunitario de la estrategia Aiepi. Serie OPS/FCH/CA/04.2 (1).E.

http://www.cruzroja.org/salud/redcamp/docs/SaludComu/Guiafacilitadores.pdf

Silva, Joana A.; Dalamaso, Ana S. W. Agente comunitário de saúde: o ser, o saber, o fazer. Rio de Janeiro: Fiocruz, 2002.

Simeão, Elmira; Oliveira-De-Melo, Cristiano. "Alfabetização em informação para a capacitação do agente comunitário de saúde no Brasil: proposta de mediação baseada no modelo extensivo e colaborativo". Reciis, 2009 , v. 3, n. 3, pp. 58-66

http://www.reciis.cict.fiocruz.br/index.php/reciis/article/view/283/329

Vázquez-Navarrete, Maria-Luisa; Ferreira-da-Silva, M. Rejane; Siqueira-Campos, Eliane; Campos-Pereira, Ana-Paula; Silva-Diniz, Alcides; Leite-Veras, Ida; Grande-De-Arruda, Ilma K. "Visión de los diferentes agentes sociales sobre la participación social en el sistema de salud en el nordeste de Brasil: una aproximación cualitativa". Revista española de salud pública, 2002 v. 76, n. 5, pp. 585-594.

\section{Aurora Cuevas-Cerveró y María-Antonia García-Mo-} reno. Universidad Complutense de Madrid. macuevas@ccinf.ucm.es mariaant@ccinf.ucm.es

El profesional de la información está indizada por la base de datos

ISI Social Science Citation Index, de Thomson Reuters, y según el Journal Citation Report ha obtenido un Factor de impacto de 0,400 